\title{
Poder y comunicación, o el discurso del (auto)elogio.
}

\section{Power and communication, or the (self)praise discourse}

\author{
Lázaro M. Bacallao Pino
}

Universidad de La Habana

bacallao@fcom.uh.cu

\section{Resumen:}

A partir de un análisis de las interrelaciones de lo comunicativo con otras esferas de la realidad social -política, económica, cultural y militar-, se indaga en las conexiones entre comunicación y relaciones de poder, así como la funcionalidad de una representación social del poder de lo mediático.

Palabras clave: medios, periodismo, comunicación, relaciones de poder.

\section{Abstract:}

Taking into account the relationships between communication and other social dimensions -such as politics, economy, culture, and military forces-analysis, the article investigate the connection between communication and power relationships, as well as the functions of a social representation of media power.

Key-words: media, journalism, communication, power relations.

\section{Introducción}

El lugar específico de los medios de comunicación al interior de las relaciones de poder generales de la sociedad, ha sido una obsesión constante de teóricos, políticos, profesionales del sector, empresarios, e incluso ha llegado a arraigarse en el imaginario popular. La metáfora de la comunicación en tanto (contra)poder - a la que han acompañado otras, como la de comunicación-información, comunicación-educación, comunicación-transporte, comunicación-tecnología -, resulta, en realidad, sumario de todas aquellas. Su expresión más generalizada sería la llamada Teoría del Cuarto Poder, sintetizada en la frase feliz del pensador conservador británico Edmund Burke, hace dos siglos, mientras contemplaba la galería de prensa de la Cámara de los Comunes: "Ahí se sienta el cuarto poder, y sus miembros son 
más importantes que todos los demás”. Desde entonces, se integró a la cultura política, y ha devenido lugar común que opera activamente en el horizonte conceptual y práctico de la información, aun cuando se maneje sin demasiada exactitud (Soria, 1994).

La certeza del poder de los media se ha apoyado, tradicionalmente, sobre el supuesto de que estos determinan la opinión pública, modelan las representaciones sociales, promueven determinadas conductas y valores afines a un modo de vida específico. Es decir, se ha argumentado a partir de su dimensión cultural y su encargo al interior de los procesos de construcción del consenso social en la modernidad. Sin embargo, desde la atalaya de la contemporaneidad, una visión compleja de las interrelaciones entre medios y dinámicas de poder, requiere de: $1^{\circ}$ ) un examen de sus enlaces multidimensionales con el resto de los diversos procesos y estructuras sociales; pero también de: $2^{\circ}$ ) un análisis que considere la funcionalidad y rentabilidad -tanto para los propios media como para los agentes sistémicos de la dominación- de una representación social que exalta el poderío de los medios.

Quizás uno de los ejemplos más evidentes de esa visualización del poder mediático, que terminó siendo funcional a la conservación sistémica en su totalidad (y a la reafirmación validante de su proceso de construcción de hegemonía) es la cobertura del escándalo Watergate realizada por The Washington Post, y la exaltación, en la construcción del mito, de la renuncia del presidente Richard Nixon, como resultado de esa labor periodística.

Una indagación en torno a la funcionalidad positiva de esa visualización del poder comunicativo ha de transitar por dos senderos complementarios: 1) los fundamentos de la modernidad capitalista en tanto régimen de visibilidad;2) el escamoteo del núcleo duro de los procesos de cambio social a los actores contrahegemónicos y sus prácticas.

La primera cuestión remite a la fundación misma del proyecto de la modernidad, y el encargo dado a la prensa como parte del mismo. En los fundamentos propuestos por JeanJacques Rousseau para la Revolución Francesa, la prensa tenía el papel fundamental de garantizar la visibilidad de toda la sociedad, de arrojar luz sobre los oscuros rincones del poder de la nobleza, garantizando buenas conductas ante la luz. Sin embargo, este propósito aparece, en su reverso -como ha analizado el pensador francés Michael Foucault-, ligado a una de las transformaciones esenciales en los mecanismos de ejercicio del poder en la modernidad. La metáfora por excelencia de tal cambio resulta el panóptico de Jeremías Betham, su obsesión por lograr una visibilidad totalmente organizada alrededor de una mirada dominadora y vigilante; una visibilidad universal, que actuaría en provecho de un poder riguroso y meticuloso -"onmicontemplativo”. La visión lírica de Rosseau, y de otros pensadores de entonces, no consideró los componentes materiales y económicos de la opinión, puerta de entrada para la distorsión de las piedras angulares -libertad y objetividad- de toda una teoría de la prensa. Al respecto, plantea Foucault (1980):

Creyeron que la opinión sería justa por naturaleza, que se extendería por sí misma, y que sería una especie de vigilancia democrática. En el fondo, es el periodismo -innovación capital del siglo XIX- el que ha puesto de manifiesto el carácter utópico de toda esta política de la mirada. 
Esa consideración de la visibilidad en tanto fundamento del funcionamiento de lo sociopolítico moderno, ha hallado su refuerzo en las concepciones postmodernas acerca de la sociedad transparente, presentada como resultante de la creciente mediatización de los diversos campos de la vida: social, económico, político, cultural, educativo. Ciertamente, la pasada centuria estuvo atravesada por una gradual centralidad de los medios en las prácticas y dinámicas sociales: si ha sido definido como el siglo de la guerra (Hobsbawm, 1998), otra metáfora pertinente para cualificarlo sería la de la comunicación. Desde el surgimiento del modelo de la gran prensa de masas -en sus albores-, hasta la finisecular explosión de las tecnologías de la información y la comunicación (TICs) -con el email y la Internet como puntas de lanza-, pasando por la aparición de la radio y la televisión, los últimos 100 años, pueden también ser considerados una cronología de lo mediático.

La indagación de las reubicaciones de lo comunicativo, como parte de tal expansión, en los resortes de la dominación, requiere en primer lugar-como se ha expresado- un examen de sus conexiones con otros campos sociales, en particular: 1) el político; 2) el económico; 3) el militar y 4) el cultural. Este análisis, con sus entrecruzamientos, complementaciones y reconfiguraciones -en sintonía con demandas y contextos históricos-, resulta la perspectiva pertinente para una comprensión compleja de las especificidades y espesor propio delos media en el concierto de los mecanismos de dominación/liberación. Una definición gramsciana de la opinión pública, resulta punto de partida adecuado, al ofrecer una conceptualización que trasciende el simple hecho de la visibilidad, y establecer sus vínculos con los procesos políticos:

Lo que se llama 'opinión pública' está estrechamente vinculado con la hegemonía política, o sea que es el punto de contacto entre la 'sociedad civil' y la 'sociedad política', entre el consenso y la fuerza. (...) La opinión pública es el contenido político de la voluntad política pública que podría ser discordante (...) (Gramsci, 1997, p 151).

De ahí que, plantee, si bien una historia de la opinión pública no puede limitarse a la modernidad -pues elementos de la misma han existido siempre-, en su sentido actual, su nacimiento se sitúa "en el período de lucha de la nueva clase burguesa por la hegemonía política y por la conquista del poder" (Gramsci, 1997, p 151). Entre los órganos de la opinión pública que se intenta monopolizar, Gramsci (1997, p 134) menciona a los periódicos, los partidos y el parlamento, pero admite -ya desde entonces- que la prensa en general resulta la parte más dinámica -aunque, reitera, no la única- de la estructura ideológica de la clase dominante.

La visión gramsciana resulta - con su esencial enriquecimiento del análisis, desde una perspectiva crítico marxiana, en torno a las dinámicas y procesos superestructurales- aporte cardinal en la comprensión de ese lugar y encargo de lo mediático en el marco de los procesos político-culturales de construcción de hegemonía. Porque, para Gramsci, lo cultural resulta dimensión esencial de lo político, y la política se comprende como labor de estructuración y desarrollo de la hegemonía de una clase sobre la sociedad, de lo cual deriva un enfoque de 
la dominación que no es solo imposición, coerción y fuerza, sino también capacidad para establecer y preservar el liderazgo intelectual y moral, para construir en torno a su proyecto un nuevo sistema de alianzas sociales (Acanda, 2002).

Tales interrelaciones entre prensa y procesos hegemónicos, resultan asimismo punto de partida para una solución de continuidad en el establecimiento de los nexos entre los media y la dimensión económica. Aun cuando los procesos hegemónicos implican "no solo objetivos económicos y políticos unificados sino también una unicidad intelectual y moral, no sólo a corto plazo sino a largo plazo" (González Casanova, 1984, p 18), ello no ha de significar una subvaloración de los procesos estructurales; pues si bien la hegemonía "es ético-política, no puede dejar de ser también económica, no puede menos que estar basada en la función decisiva que el grupo dirigente ejerce en el núcleo rector de la actividad económica" (Gramsci en Acanda, 2002, p 275).

La ubicación de lo mediático en la trama de los procesos hegemónicos, establece una integración de aquel a las diversas dimensiones de estos, siempre en niveles heterogéneos y formas múltiples. Una singularidad de los media, señalada igualmente por Gramsci (1997, p 72), complejiza aún más dichas articulaciones. Estos resultan instrumentos técnicos que poseen una doble fenomenología: son, al mismo tiempo, estructura y superestructura. A la vez que objeto de propiedad, son elementos inherentes del hecho ideológico.

El proceso, de acuerdo con el análisis gramsciano, transcurre en este sentido: un determinado grupo hegemónico, como parte de su propósito de lucha por el poder y su conservación, crea determinadas superestructuras que, a su vez, determinan la formación de una "estructura material especial" para su difusión. Sin embargo, el desarrollo de tales superestructuras, no es inmanente a su esa particular estructura material, sino a la general social. "Lógicamente y también cronológicamente se tiene: estructura social-superestructuraestructura material de la superestructura" (Gramsci, 1997, p 73).

Pero mientras desde sus inicios y hasta las últimas décadas del siglo XX, la generalidad de los estudios y conceptualizaciones en torno a los medios hizo énfasis en la dimensión político-cultural de su vinculación a los procesos hegemónicos, la progresiva concentración del sector -cuya expresión final son los grandes grupos transnacionales de la comunicación-, a la par de la sucesiva penetración de los capitales de otra ramas (electricidad, agua, aeronáutica) en el mismo - con su secuela más terrible en la brutal comercialización de las dinámicas y producción mediáticas-, ha llevado a un mayor detenimiento en sus nexos con la dimensión económica.

Este tránsito ha sido sintetizado, recurriendo a la conocida clasificación althusseriana, como el paso de los media de una condición de "aparatos ideológicos del Estado" a otra, de "aparatos ideológicos del Mercado". Pero, de hecho, lo comunicativo no solo resulta un sector que coadyuva, desde lo cultural, al sometimiento a un orden dominador en la economía y, por tanto, es garante de la generación de plusvalía; sino que se ha convertido en un sector económicamente atractivo en sí mismo. La comprensión de ese interés por el sector 
comunicacional, y la colonización extrema del mismo por la racionalidad económica del capital, requiere ser contextualizado.

En primer lugar, es parte de la tendencia inherente al capital de totalización de su dominio sobre todas las esferas de la vida y de mundialización de la misma. Los teóricos críticos de Frankfurt, para dar cuenta de ese proceso de invasión del campo cultural por las lógicas de la producción moderna, acudieron al concepto de industria cultural. Pero, al mismo tiempo, este fenómeno aparece ligado al desarrollo espectacular de las TICs. También debemos a un frankfurtiano, Herbert Marcuse (1968), un examen -en tono apocalíptico- de la racionalidad instrumental que acompañaría, de forma inherente, a la racionalidad tecnológica capitalista, en un proyecto absoluto del cual resultaría imposible escapar para fundar aquel espacio históricamente trascendente del cambio social revolucionario, previsto por los fundadores del marxismo.

Un análisis de las conexiones entre las tecnologías comunicativas y las relaciones de poder, resulta imprescindible en una indagación acerca de los lazos entre comunicación y poder. De hecho (y la siguiente afirmación no debe entenderse en un sentido determinista), tal dimensión tecnológica cruza de manera transversal y conecta, dando coherencia, a la vinculación entre lo comunicativo y el resto de los campos de las relaciones de poder mencionados con anterioridad.

En correspondencia con el hecho de que todo modo de producción no es sólo una manera de producir los objetos, sino un modo de vida (Marx y Engels, 1969), toda técnica productiva tiene, en cierto grado y modalidad, una determinada cualidad y función superestructural; por tanto, pudiese ser considerada potencialmente, según ciertos criterios, una técnica disciplinaria (De Gaudemar, 1991). La propia organización productiva en que se utiliza esa técnica, como se ha mostrado en los exámenes de la arquitectura y distribución en la fábrica moderna $-\mathrm{y}$ en otras instituciones sociales, como las cárceles y hospitales-, aparece diseñada y dispuesta de manera tal que facilite las comunicaciones favorecedoras y agilizantes del proceso productivo, mientras trata de impedir aquellos canales $\mathrm{y} / \mathrm{o}$ vías que puedan resultar "subversivos" (Foucault, 1997).

Las tecnologías comunicativas, dada su propia naturaleza, habrían seguido un camino "inverso" al de las técnicas originariamente productivas, en el sentido siguiente: de herramientas con un propósito primariamente superestructural, han pasado a ser artefactos disciplinarios y, al mismo tiempo, productivos. Esto, no sólo a partir de la comercialización del sector mediático global, sino a través del empleo creciente de las TICs -hasta convertirse en elementos imprescindibles-, en el funcionamiento de la actividad económica, y, muy especialmente, sus mecanismos financieros globales. El lugar específico que ha llegado a ocupar la comunicación y la información en los engranajes del sistema productivo capitalista, ha sido descrito por Castells (1999, p 95) con la afirmación de que las TICs, en la época del informacionalismo, "le dan otra vuelta de tuerca al modelo clásico de ganancia capitalista".

Esto confirma las teorizaciones gramscianas acerca de las conexiones entre la estructura e infraestructura específicas de la superestructura y la estructura/infraestructura general social. 
Pero, además, evidencia la especificidad de los roles de lo comunicativo en la configuración e implementación de los procesos sistémicos del capitalismo, en tanto régimen cuyo espíritu universal encuentra el soporte tecnológico más pertinente para la consumación de esa vocación, precisamente en los artefactos comunicativos.

Otra de las interrelaciones de singular interés a los efectos de la indagación en los vínculos comunicación/poder, resulta de los lazos entre las tecnologías comunicativas y la guerra. ${ }^{1} \mathrm{De}$ hecho, existe una estrecha conexión -en varios sentidos- entre los avances de lo bélico y lo comunicativo, a lo largo del siglo XX. De forma general, muchas de las nuevas tecnologías comunicativas desarrolladas en el período han tenido su génesis y primeros usos prácticos en el sector militar. El ejemplo más reciente de ello lo constituye ARPANET -proyecto desarrollado por el Departamento de Defensa de Estados Unidos (EE.UU) a través de su Agencia de Proyectos de Investigación Avanzados (ARPA, por sus siglas en inglés) en 1969-, considerada primer antecedente de Internet.

Pero estas conexiones tienen expresiones más significativas, en el terreno de lo cultural y simbólico. A partir del análisis de varios acontecimientos ocurridos entre las décadas de 1960 y 1970 -el estreno por la fuerza aérea de los EE.UU, en 1972, de una bomba teledirigida que llevaba en la nariz una cámara de televisión, cuyo nombre Maverick Missile, fue tomado de una serie de televisión; o que los aparatos de aviación norteamericanos durante la guerra de Vietnam o en Bolivia, pintaran en sus alas victoriosas al Pato Donald-, Mattelart (1989) examina las relaciones entre realidad/ficción, en las cuales, la superposición de la segunda sobre la primera, tiene lugar a partir de una "transferencia semántica" de la cultura de masas a los campos de batalla.

En realidad, cada nueva técnica, como afirmara Marshall McLuhan, ha necesitado de una guerra para ganar la validez práctica, y los dispositivos comunicativos están lejos de constituir la excepción. De hecho, cada nuevo media, ha tenido en una particular contienda bélica, el espacio propicio para su validación. Las tecnologías comunicacionales han presentado un rol específico tanto al interior del funcionamiento de la propia organización y estructuras militares, como en la labor informativa y propagandística en torno a los conflictos armados.

El extremo, en el empleo de los artefactos comunicativas para la guerra, sería la invención de armas cuyo objetivo no es provocar las tradicionales lesiones y heridas, sino, por ejemplo, generar un ruido capaz de producir dolores de cabeza, pánico o sordera, como elllamado Long Range Acoustic Device (LRAD), cuya introducción y empleo por las fuerzas norteamericanas desplazadas en Iraq, fue anunciado en marzo de 2004. Tal artilugio, según se explicaba, se emplearía probablemente contra manifestaciones, en puestos de controly desalojo de edificios (Europa Press, 9 marzo, 2004).

1. Para un análisis detallado de tales nexos, puede consultarse, del propio autor: "La comunicación de la guerra/ la guerra de la comunicación: disturbios y convergencias”, Revista Punto Cero, nro. 10, 2004. Universidad Católica Boliviana San Pablo, Bolivia. pp. 32-41. 
La funcionalidad informativo-propagandística de los media en cada uno de los principales enfrentamientos armados del siglo XX -desde la guerra hispano-cubano-norteamericana (temporalmente ubicada en la centuria anterior, pero bisagra en el tránsito hacia el propósito de los conflictos bélicos en el nuevo siglo)-, se sintetiza en una cronología de constantes reconfiguraciones, a partir de los contextos específicos, la llegada de nuevos lenguajes al campo mediático, los propósitos y características de cada conflagración. Desde el empleo de cierto armamento con fines psicológicos -el llamado "bombardeo moral", implementado durante la II Guerra Mundial-, el cual supone una casi exclusiva dimensión propagandística (desmoralizante, mediante la estrategia de Ermattungstrategie (agotamiento) (Ash, 1999)) del hecho destructivo, hasta las distorsiones periodísticas acerca de los sucesos en el campo de batalla -y sus consiguientes crisis de credibilidad de la prensa-, esta cuestión ha sido cardinal.

Los procesos de subinformación y desinformación asociados a las guerras contemporáneas, han sido analizados ampliamente, sobre todo en los conflictos de los últimos veinte años. Incluso, durante la segunda mitad del siglo XX, el mundo asistió (¿asiste?) a una hostilidad caracterizada por su naturaleza esencialmente cultural -en su sentido gramsciano que incluye lo ideológico y político-, la llamada Guerra Fría y los conflictos de baja intensidad -enfrentamientos, básicamente, propagandístico-mediáticos. Pero, a finales de la pasada década, se ha llegado al extremo de considerar al poder militar en una función garante de la agresión cultural. "El papel que desempeñarán en realidad las fuerzas armadas estadounidenses será el de mantener el mundo seguro para nuestra economía y abierto a nuestro asalto cultural", declaraba el comandante Ralph Peters (en George, 2002), de la Oficina del Jefe Adjunto del Estado Mayor para la inteligencia del Ejército de los EE.UU, en 1997.

Pero, al mismo tiempo, el sector militar ha servido de antecedente habitual para el desarrollo de tecnologías de la comunicación, cuya utilización luego se ha extendido hacia el ámbito comercial, en una expresión de la armonía entre el complejo militar-industrial y la economía capitalista. Al examinar esa convergencia y sus implicaciones para el sistema y su economía política, hace cuatro décadas, Che Guevara (2006, p 13) calificaba los aparatos de la defensa y producción en el capitalismo como "negocios gemelos y todos los grandes adelantos obtenidos en la ciencia de la guerra pasan inmediatamente a la tecnología de la paz ( ... )”.

En síntesis, estaríamos frente a una doble rentabilidad -económica y cultural- de lo mediático y sus tecnologías, en su interrelación con el campo militar; que, a su vez, conecta con idénticas racionalidades en su inserción en el resto de las esferas sociales. Dos particularidades distinguen a las tecnologías comunicativas -y, luego, al hecho comunicacional mismo- en su vinculación con esas dos dimensiones generales esenciales de los procesos hegemónicos:

Primero, el espíritu de universalidad, tanto en su sentido geográfico como societal, que acompaña de manera inherente a lo comunicativo. Su empeño, al menos en la versión más sublime, radicaría -tal como confirma el análisis etimológico del término mismo-, en el propósito de la articulación y el intercambio, más allá de las fronteras que el espacio y el tiempo colocan a la acción humana. Pero, a la vez, la vida en sociedad resulta inherentemente 
comunicativa, pues el contrato social supone la configuración de unos procesos organizativos y culturales, de interrelación entre la dimensión individual y social de los sujetos, de establecimiento de unos vínculos y relaciones sociales que tienen lugar en todas las esferas de la vida, pero que presentan como complemento y recurso de expresión la dimensión comunicativa.

Esta arista comunicativa de la cuasi totalidad de los campos de la vida social ha sido profusamente analizada, sobre todo a partir del boom de lo comunicacional ocurrido en las últimas décadas. Sin embargo, en ocasiones esto ha conducido a extremos en las valoraciones acerca del lugar y el espesor de esa dimensión al interior del resto de los campos sociales. El ejemplo teórico más evidente de ello sería el denominado pancomunicacionismo.

Segundo, la singularidad de las tecnologías comunicativas -ya esbozada- de presentar una condición original substancialmente disciplinario-cultural. Tal cualidad, en el resto delastécnicas los procesos industriales de la modernidad, resulta más ambigua o difícil de visualizar. E, incluso, hasta más cuestionable su presencia, pues - como se ha apuntado- la afirmación gaudemariana acerca de la condición disciplinaria de toda técnica productiva, conduce, de manera peligrosa, al determinismo de asumir la existencia de una inherente racionalidad dominadora en la técnica, imposible de ser separada de aquella y, por tanto, de sus usos sociales.

Las tecnologías de la comunicación, al poseer esa cualidad cultural primaria, y, luego, también una funcionalidad económica -acrecentada en el tiempo-, devienen herramientas atractivas tanto en sí mismas, como en el propósito de complementar esa dimensión disciplinaria del resto de las técnicas y formas organizativas de la producción. En una dinámica sistémica cuya tendencia es, cada día más, a la exclusión de los sujetos y, por tanto, a su desvinculación del área productiva, se requiere de recursos de "conexión light" que mantengan esas ligaduras necesarias para los procesos hegemónicos, produzcan falsos sentidos de participación y pertenencia, y minimicen la posibilidad de creación de espacios out, gestantes de proyectos de cambio social antagónicos.

Tal pertinencia de la comunicación en tanto elemento coadyuvante a la coherencia del sistema, como recurso reticular de articulación de las distintas esferas -tanto intra como intercampos societales-, a partir de ser una de las estrías a través de las cuales transita el intercambio (input/output) entre los subsistemas y de estos con el sistema social, conduce a la necesidad de un análisis acerca de la oportunidad y beneficios de una representación social del poder mediático, en la sociedad contemporánea.

En primer lugar, la propia sociedad se ha pretendido definir desde lo comunicativo conceptos como los de sociedad transparente, toman como fundamento su dimensión comunicacional. Pero una conceptualización que asume como centro este eje, escamotea no solo una comprensión integral de los procesos sociales, sino determinados núcleos esenciales de las prácticas y estructuras de los sistemas sociales. La disolución igualadora de la condición social en la cualidad comunicativa, conlleva a tendencias y consecuencias múltiples, todas vinculadas con la ubicación de esta dimensión en el entramado de las relaciones sociales de poder. 
A nivel del imaginario común $-y$ sin que, necesariamente, acontezca lo mismo en la realidad- se percibiría una suerte de "transferencia de poder" desde otras áreas de la sociedad hacia la esfera mediática, en un proceso que se ve favorecido por la propia visibilidad inherente a los media, así como por los antecedentes de la presencia, en el sentido común, de una imagen poderosa de aquellos. El tema del "poder" de los medios - estrechamente ligado a la cuestión de sus efectos-, ha permanecido como objeto de indagación y teorización, casi de manera obsesiva, en los discursos acerca de estos y su encargo social.

Una revisión en la cronología de los estudios y conceptualizaciones sobre los medios, muestra esa constancia; subyacente incluso, en ocasiones, en aquellas posturas teóricas más alejadas de las visiones omnipotentes sobre los medios, en términos de "influencias", "influjos" o "importancia” mediáticas. Aun en investigaciones y teorías que se apoyan en la perspectiva de las mediaciones, se han asumido enfoques que conducen a concepciones de lo mediático desde una centralidad tal, que termina siendo una suerte de mediación "fundamental", o "mediación mediada”. Se traiciona así el mejor espíritu de la dialéctica mediacional, en su sentido de análisis multirrelacional de los entrecruzamientos constantes de las diversas dimensiones de la realidad -con diferencias siempre coyunturales de espesor entre cada una de ellas, dadas por las circunstancias contextuales históricas-, por los cuales transitan los procesos sociales.

El discernimiento del lugar específico de lo cultural en las dinámicas de continuidad y cambio social, ha sido asunto controversial, llevado habitualmente a los extremos opuestos de los determinismos economicistas y culturalistas. Fronteras estas que marcan los límites de los análisis posibles acerca del "poder" de lo cultural, localizándolos entre las dos visiones antagónicas de la nulidad o absolutidad del mismo. Ambos puntos de vista han estado presentes en la historia del pensamiento crítico contemporáneo, a pesar de que, en los fundadores del marxismo, se encuentran criterios reprobatorios acerca de cierta tendencia en sus propios exámenes a ponderar la dimensión estructural y soslayar la superestructural. En una revisión notable de esa inclinación, se explicaba que considerar el factor económico como el único determinante en la historia de la producción y la reproducción de la vida real, resultaría una tergiversación del planteamiento del marxista, convirtiéndolo en "una frase vacua, abstracta, absurda” (Engels, 1969, p 748).

Mostrar las conexiones entre la dimensión comunicativa y otros campos especialmente significativos de las relaciones de poder, por tanto, contribuye a dilucidar con mayor certeza el espacio de la comunicación, desde sus especificidades al interior de las estructuras y procesos superestructurales, en la conformación de la dominación -y, en consecuencia, de la liberación. Se trata de, frente a una perspectiva que pretende examinar la centralidad en sí misma de la dimensión comunicativa en las relaciones de poder - hasta proponer una dictadura comunicativa-, explorar su centralidad articulante - dadas sus singularidades mencionadasentre las distintas dimensiones de los procesos de configuración de hegemonía.

Es decir, analizar lo comunicativo como recurso para, entre otros fines:

1) la dinamización de los mecanismos económicos, en una época de constantes amenazas 
sobre la llegada al límite de las posibilidades de producción y reproducción del sistema, y sus fuerzas productivas;

2) la relegitimación de lo político en tiempos de crisis de legitimidad de las estructuras y organizaciones políticas tradicionales (partidos) y la emergencia de nuevas formas de militancia y acción social organizada;

3) la generación de un simulacro de sentido de vinculación/pertenencia, a partir de la difusión socializante de simbologías, patrones y normas de conducta, estilos de vida y representaciones de la realidad;

4) el perfeccionamiento de unos instrumentos de violencia y represión (militares), con el propósito de garantizar una mayor seguridad de los fuerzas propias, así como de "higienizar" las acciones de destrucción y muerte, tanto a los ojos de sus ejecutantes como ante la mirada del mundo, creando tecnologías que incrementen la distancia entre el victimario y sus víctimas, a la vez que promoviendo un clima de opinión favorable a determinada agresión, a partir de supuestos argumentos justificantes.

Una ideología de la comunicación que obvie tales complejidades y pretenda solo una visualización del poder de la visibilidad -o una visualización del poder de la ocultación y la manipulación- en su manifestación de los resortes mediáticos, no solo conduciría a una visibilidad desenfocante del resto de las dimensiones de la dominación. Al mismo tiempo, hurtaría también las posibilidades de comprensión crítica -y, por ende, transformadora- de una realidad cuyos niveles de complejidad e imprevisibilidad se han visto incrementados de manera significativa justamente a partir de la aparición de múltiples canales de flujo de comunicación e información, que han dado lugar a nuevos tipos de intercontectividad e indeterminación en el mundo, los cuales aún no conocemos en toda su multiplicidad de implicaciones (Thompson, 1998).

Un discurso de autorreferencialidad, en torno al poderylosmedia, no solohacemás atractivo al sector y contribuye a desenfocar otras dimensiones de las relaciones de dominación, sino que, además, coadyuva a atenuar la propia visión del poder, toda vez que resulta en un recurso de autolegitimidad de un mecanismo de dominio que, supuestamente, se fundamentaría en el principio democrático de la "visibilidad" de ese poder, cuando en realidad resulta en una visibilidad desarticulante de la totalidad social -continuación del espíritu fragmentario del proyecto de la modernidad, llevado a sus extremos en los principios postmodernos- $y$, por tanto, funcional a una opresión que se afinca, precisamente, en su naturaleza total y universal.

La comprensión de esa visibilización de un poder de la visibilidad, también requiere ser contextualizada en los reordenamientos acontecidos en los dispositivos de la censura-desde el defecto hasta el exceso (Ramonet, 1998)- en los últimos tiempos. Esa representación social que exalta el poderío de los medios, puede dar otra vuelta de tuerca a las prácticas de censura por abundancia, en sintonía con el resorte de la "tolerancia represiva" -en términos de Marcuse (en Eco, 2003)-, según el cual, demostrada la propia falta de prejuicios autoflagelatoria, los medios ya no se interesarían en reformarse. 
Estaríamos además, con esta postura, frente el completamiento de un mecanismo de desestructuración de los procesos analíticos de la dominación, que complementa la conformación de una "extraña dictadura" - según la definición de Vivianne Forrester (1997)-, caracterizada por ser un esquema de dominio inexorable y global, pero a la vez intangible, el cual no remite a una estructura o una institución específica (visible) encargada de gobernar.

La visión del poderío excluyente, absoluto y único de la comunicación, contribuye sobremanera a la legitimación de ese supuesto paradigma inasible y fluido -como lo comunicativo mismo-, imposible de ser tomado, de los resortes de la dominación. Pretender presentar las relaciones comunicativas y sus interrelaciones con el resto de los dimensiones del poder, sus estructuras y relaciones, como una "indagación general sobre el poder" y no al interior de un entorno que contempla las cuestiones políticas, económicas, tecnológicas, culturales y militares, resultará desacertado para cualquier empeño crítico.

La respuesta a tales pretensiones, estará en desentrañar el espesor específico de lo comunicativo al interior de los actuales procesos hegemónicos -en sus distintos niveles, desde lo global hasta lo local-, en sus interconexiones con el resto de sus dimensiones. Acudiendo al concepto de deconstrucción exteriorizante, propuesto por Briankle G. Chang (2000, p 19), se trata de realizar una:

crítica historizante porque se logra dispersando el momento aislado de comprensión como asunto hermenéutico en el conjunto, plagado de conflictos, de la heteroglasia social, haciendo explotar con ello la unidad nodal de comunicación en las prácticas interdiscursivas que constituyen la estructura social general", [exteriorizando el] "microevento de la comunicación a su macroestructura de determinación.

En tiempos en que incluso buena parte de las teorizaciones en torno al cambio social que vivimos, se fundamentan en las experiencias mediáticas de los propios autores (Jameson, 2002), solo a partir de un análisis de tal naturaleza, se podrá comprender la especificidad de lo comunicativo, en sus múltiples solapamientos con el resto de los campos sociales. Ello permitirá, además, dilucidar las intrincadas sutilezas y peculiaridades de la fetichización de la mercancía información -y, en especial, de sus resortes de autorreferencialidad-, como parte de la concentración de la industria mediática, en sus conexiones con el doble fetichismo actual de la producción y del consumidor - por intermedio de los mediapaisajes (Appadurai, 2002)-, y como parte de la trama del desarrollo y perfeccionamiento de la "economía del poder" (Foucault) que caracteriza al proyecto de la modernidad capitalista. 


\section{Referencias bibliográficas}

Acanda González, J. L. (2002). Sociedad civil y hegemonía. La Habana: Centro de Investigación y Desarrollo de la Cultura Cubana Juan Marinello.

Appadurai, A. (2002). "Disyunción y diferencia en la economía cultural global”. Revista Criterios № 33. pp. 13-41.

Ash, E. (1999). “Terror Targeting: The Morale of the Store”. Aerospace Power Journal Vol. XIII No 4. Obtenido el 4 de marzo de 2008 en

http://www.airpower.maxwell.af.mil/airchronicles/apj/apj99/win99/ash.htm

Traducción disponible en http://www.europa1939.com/documentos/terror.html.

Castells, M. (1999). La era de la información. Vol. I. Madrid: Alianza Editorial (2a ed.).

Chang, B. G. (jun. 2000). "La desconstrucción de la comunicación: Derrida y la (im)posibilidad de la comunicación”. Revistas Temas No 20/21.pp. 18-29.

De Gaudemar, J. P. (1991). "Preliminares para una genealogía de las formas de disciplina en el proceso capitalista de trabajo”. En: Espacios de poder. Trad. J. Varela y F. Alvarez-Uría. Barcelona: La Piqueta.

Eco, U. (23.02.2003). “Crítica al Periodismo”. Avizora. Obtenido el 20 de febrero de 2008 en http:// www.avizora.com/publicaciones/comunicacion/textos/critica_al_periodismo.htm.

Engels, F. (1969). “Carta a J. Bloch”. Obras escogidas. Tm. I. Moscú: Progreso. pp. 748-49.

Forrester, V. (1997). El horror económico. México: Fondo de Cultura Económica. Obtenido el 3 de marzo de 2003 en http://lanic.utexas.edu/ sela/AA2K/ES/cap/N50/rcap5016.htm.

Foucault, M. (1980). “El ojo del poder”. Entrevista. En: El Panóptico. Trad. J. Varela y F. Alvarez-Uría. Barcelona: La Piqueta.

-(1997). Vigilar y castigar: nacimiento de la prisión. México: Siglo XXI Editores.

George, S. (2002). Informe Lugano. La Habana: Editorial Ciencias Sociales.

González Casanova, P. (1984). La hegemonía del pueblo y la lucha centroamericana. Costa Rica: Editorial Universitaria Centroamericana.

Gramsci, A. (1997). Gramsci y la filosofía de la praxis. Comp. G. Ramos y J. L. Acanda González. La Habana: Editorial Ciencias Sociales.

Guevara, E. (2006). Apuntes críticos a la economía politica. La Habana: Ocean Sur.

Hobsbawn, E. (1998). Historia del siglo XX. Tm. I. Buenos Aires: Grijalbo Mondadori.

Jameson, F. (2002). “Notas sobre la globalización como cuestión filosófica”. Revista Criterios № 33. pp. 42-69.

Marcuse, H. (1968). El hombre unidimensional. Ensayo sobre la ideología de la sociedad industrial avanzada. La Habana: Instituto del Libro.

Marx, C. y Engels, F. (1969). “La ideología alemana”. Obras escogidas. Tm. I. Moscú: Progreso.

Mattelart, A. (1989). La cultura como empresa multinacional. México: Editorial Era.

Ramonet, I. (1998). La tiranía de la comunicación. Madrid: Editorial Debate S.A.

Soria, C. (ene. 1994). "Las relaciones entre la información y el poder político: revisión crítica de la teoría del cuarto poder”. Diálogos No 38. pp. 23-32.

Thompson, J. B. (1998). Los media y la modernidad: una teoría de los medios de comunicación. Barcelona: Paidos. 